\title{
Quantitative Methodology Research: Is it on Psychologists' Reading Lists?
}

\author{
Laura Mills, Eva Abdulla \& Robert A. Cribbie \\ York University
}

Two studies investigated the extent to which researchers are accessing quantitative methodology publications. The first study investigated the number of references to quantitative methodology sources in research articles from six prominent psychology journals. The analyses revealed that $39 \%$ of all articles reviewed did not include a quantitative reference of any kind and that $72 \%$ contained two or fewer. The second study targeted publications in quantitative methodology journals to determine the frequency with which they were being referenced in non-quantitative publications and other quantitative methodology publications. Results indicate that quantitative methodology articles are being referenced equally by non-quantitative and quantitative methodology researchers, but more importantly, that the number of references to quantitative methodology articles is very low. The results of these studies suggest that researchers are diligent in determining research protocol, procedures, and best practices within their own field, but that researchers are not frequently accessing the quantitative methodology literature to determine the best way to analyze their data. Alternatively, researchers might indeed invest time into determining recent and best statistical procedures, but do not indicate so in the reference section of their work; if this is the case then this paper should be a strong reminder to psychologists about referencing the statistical approaches they utilize.

Over the past few decades, there has been a significant number of advances in data analytic methods for psychological data. These range from advanced quantitative methods for dealing with increasingly sophisticated research questions, to modern robust statistics that improve the nature of analyses when assumptions are violated, outliers are present, data are missing, etc. It is important for psychologists to be aware of, and utilize, advances in quantitative methods that are relevant to their hypotheses, as these advanced methods improve the likelihood that the conclusions from their investigations are meaningful and accurate. Recently, APA's 'Task Force on Statistical Inference' (TFSI) recommended changes in current trends for conducting, analyzing, and reporting on psychology studies. For example, researchers are encouraged to include a clear explanation of the study design, target population, and sampling procedures (Wilkinson \& TFSI, 1999). Further, the report stresses the importance of selecting an analytic method that specifically addresses the research question, and encourages researchers to be cognizant of assumption violation, multiplicities, and other potentially problematic data conditions. However, despite pleas for researchers to utilize improved quantitative methods from TFSI and many others, most continue to use conventional methods for the sake of familiarity and comfort (Keselman et al., 1998). This is extremely troubling because traditional methods are often biased or less powerful than modern analytic methods (see Wilcox, 2002). Further, recent research demonstrates that it may be a long time before the recommendations of TFSI start to manifest in psychological research publications (Cumming et al., 2007).

One possible reason why researchers are unfamiliar with advances in quantitative methods is that they are inundated with new research from within their immediate field of 
expertise, what Adair and Vohra (2003) call a 'knowledge explosion'. In essence, it is difficult to keep up with advances in peripheral fields, such as quantitative methods, when research time is being utilized just trying to keep up with advances in a specific field of specialization. Adair and Vohra quantified this knowledge explosion by showing that five prominent Psychology journals had increases in total references per article ranging from 3.1 to 8 times over 16 years. For example, from 1972 to 1998, the number of references in the Journal of Personality and Social Psychology (JPSP) tripled and those in Personality and Social Psychology Bulletin experienced an eight fold increase. Similarly, Reis and Stiller (1992) found a three fold increase in the number of articles in JPSP over three decades. This trend was foreseen by Thorngate (1990) who noted that the number of areas within psychology has grown beyond the capacity for researchers to attend to more than their own interests. He hypothesized that researchers would choose to read with 'tunnel vision', opting for summaries of work within their area, and often only works by well known authors.

The goal of research in quantitative methods for psychology is to derive, evaluate and compare available procedures for addressing the research hypotheses of psychologists. In other words, quantitative methodology research helps to improve the nature of the analyses being conducted by psychologists. Commonly these contributions are found in psychology journals that pertain to quantitative methods (e.g., Psychological Methods), and the hope is that researchers are reviewing the literature that addresses issues relevant to their research. However, as discussed above, it is unclear whether the research being conducted by quantitative methodologists in psychology is being utilized by researchers in psychology.

Therefore, the current study seeks to answer two important questions: 1) How often do published articles in psychology journals reference quantitative methodology sources? and 2) How often are articles that are published in quantitative methodology journals referenced by researchers? The first question essentially asks whether researchers conducting psychological studies are utilizing (and referencing) quantitative methodology research. The second question asks about the frequency with which published quantitative methodology articles are being referenced by non-quantitative researchers and by other quantitative methodology researchers. For both questions, we expect the number of references to quantitative methodology sources to be very low because: 1) The continually expanding amount of literature in substantive areas of psychological research limits the amount of time researchers have to devote to quantitative methodology; and
2) Previous reviews of the data analytic practices of psychological researchers have indicated that researchers continue to adopt traditional and familiar (but often inappropriate) procedures even when improved procedures are available

\section{Study One}

The purpose of the first study was to determine the extent to which journal articles include references to quantitative methodology literature. Six top-tier, peerreviewed journals were chosen: JPSP, Psychological Bulletin (PB), Journal of Consulting and Clinical Psychology (JCCP), International Journal of Psychophysiology (IJOP), Child Development (CD), and Journal of Applied Psychology (JAP). These journals were selected in an effort to represent each of the major substantive research areas of psychology.

Each reference section for every article in the 2005 and 2006 publication years in these journals was examined, with the observations of interest being the number of references to quantitative methodology and non-quantitative methodology sources. A quantitative source was defined as a reference in the form of an article, text, text section, or 'in press' manuscript with a focus on the statistical methods or procedures for analyzing data. This excluded research methodology, measurement issues, editorial comments, letters to editors, or replies to previously published articles. A non-quantitative source was defined as any other reference that was not quantitative in nature. In instances where there was ambiguity regarding the nature of a reference, the classification was determined jointly among the authors.

\section{Results and Discussion}

Of the 1161 articles from psychological journals, 12 focused on issues dealing with quantitative methodology. Six of these were in JAP, three in JCCP, two in JPSP, and one in CD. Neither PB nor IJOP published a quantitative article over this two year span. For these quantitative articles, the mean number of total references was $39.42(\mathrm{~s}=17.36)$, with a median of 36.5. The mean number of quantitative references was $16.4(\mathrm{~s}=8.8)$. These quantitative methods articles were omitted from any further analyses as the purpose of this study was to explore the referencing of quantitative methodology sources in non-quantitative psychological studies.

\section{Frequency of Quantitative and Non-Quantitative References}

For the remaining 1149 articles, the number of quantitative and non-quantitative references was tabulated. The frequency distributions of the number of quantitative and non-quantitative references from the six psychology 
Figure 1. (a) Frequency of references to non-quantitative research sources; (b) Frequency of references to quantitative methodology sources.

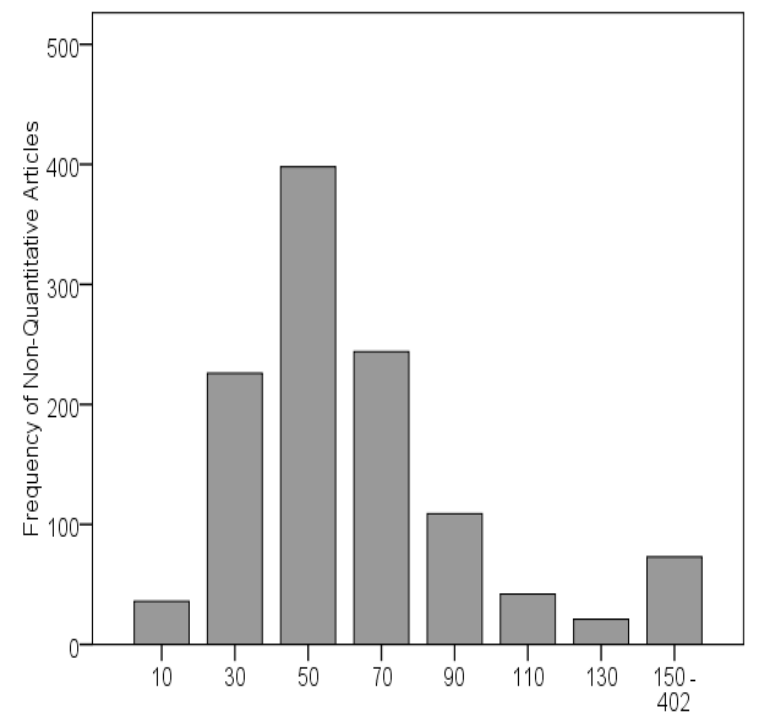

Number of Non-Quantitative References

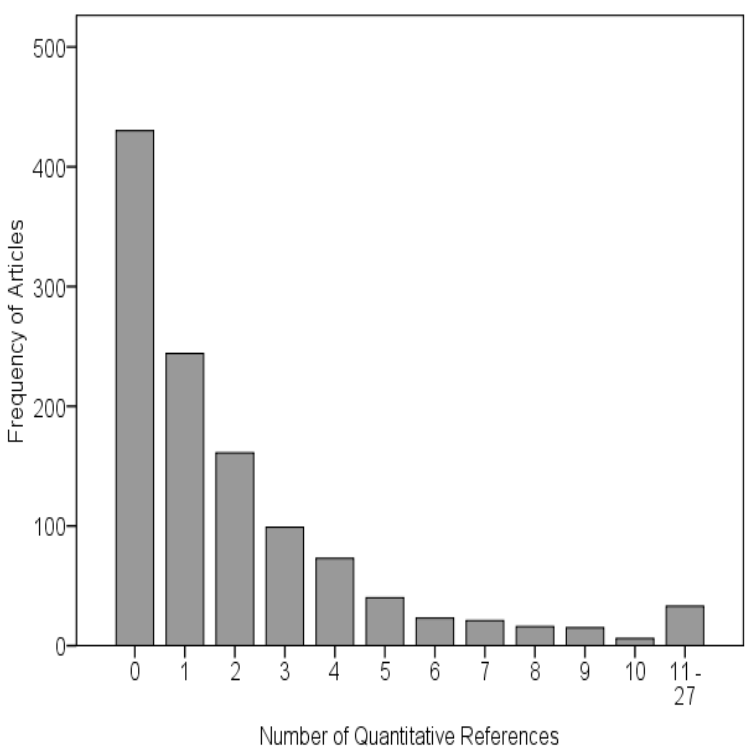

non-quantitative articles have two or fewer references to quantitative methods sources.

In terms of the proportion of references to quantitative methodology sources relative to non-quantitative methodology sources, the mean proportion was $3.64 \%$ ( $\mathrm{s}=$ .056 ) and the median proportion was $1.90 \%$. The six journals had a range of mean proportions of 1.2 to $5.2 \%$ (median proportions ranged from $0-3.8 \%$ ). In other words, a very small proportion of the references in these journals were quantitative in nature.

A paired samples t-test was used to determine whether there were differences between the number of quantitative and non-quantitative references, an appropriate test because the distribution of the difference scores was approximately normal (Zumbo \& Jennings, 2002). There was a large $\left(\eta^{2}=\right.$ 0.65) difference between the number of quantitative and non-quantitative references $(t(1148)=44.39, \mathrm{p}<.001)$. This difference is exemplified by the differences between the modes and medians for both quantitative and non-

Table 1. Measures of central tendency for the number of quantitative and non-quantitative references in applied studies.

\begin{tabular}{lllll}
\hline Reference Type & Mode & Median & Mean & Trim Mean \\
\hline & & & & \\
Quantitative & 0 & 1 & 2.2 & 1.7 \\
Non-quantitative & 43 & 54 & 65.7 & 58.8 \\
& & & & \\
\hline
\end{tabular}

Note: Trim Mean $=5 \%$ Trimmed Mean . 
quantitative references, with a modal number of quantitative references of zero and a modal number of nonquantitative references of 43 . Likewise, the medians were very disparate, with medians of 1 and 54 for quantitative and non-quantitative sources, respectively

In summary, Study 1 found that the number of references to quantitative methodology literature was low, and the overall proportion of quantitative methodology references, relative to non-quantitative references, was also low. The fact that the modal number of references was zero further points to an apparent lack of utilization of quantitative methodology sources when conducting psychological research.

These results suggest one of two scenarios: 1) Researchers, for the most part, do not diligently seek statistical methodology that will address their data issues appropriately; or 2) Researchers are indeed diligent in finding appropriate quantitative methods but do not reference appropriately. The first scenario seems most likely; it would indicate that researchers are using comfortable and familiar techniques while allocating the bulk of their research energy to designing, conducting, and writing up studies within their own specialization, a supposition supported by the findings of the frequency of cited nonquantitative sources. A counter-argument to this scenario is that researchers do gather a sense of the landscape of their data and decide that popular procedures will suffice. However, this is highly unlikely given that most studies contain data issues that render most familiar procedures invalid (Bradley, 1977; Erceg-Hurn \& Mirosevich, 2008; Golinski \& Cribbie, 2009; Keselman et al., 1998; Micceri, 1989).

The second scenario, that researchers are just not diligent in referencing the statistical methods they adopt, seems much less likely. Psychology researchers are well-versed in APA standards of referencing and it is unlikely that they would have gone to the trouble of sourcing out novel ways of analyzing data without then using the proper reference format. Indeed, using novel procedures might be perceived as a practice deserving of merit and so it would seem that researchers would be eager to cite the results of their efforts. Support for this contention comes from the finding that researchers rate citations to their research methods and data analyses as more important than citations to background theory or discussion topics (Safer \& Tang, 2009).

Another interesting question that arises from these results, as suggested by an anonymous reviewer of an earlier draft of this paper, is whether there is a relationship between the number of authors on a paper and the likelihood of including references to quantitative methodology sources. This relationship was investigated and indeed, a greater number of authors on a paper predicted higher frequencies of references to quantitative methodology literature $(\mathrm{F}(1140)=3.98, \mathrm{p}=.046)$. However, the magnitude of this relationship was small; very little of the variance in the number of quantitative methodology was explained by the number of authors $\left(\mathrm{R}^{2}=.003\right)$.

To summarize, regardless of how simple or sophisticated the research questions of a study happen to be, it is important that psychological researchers investigate the most appropriate methodology for analyzing their results. Under such a model, it is expected that the research that is directed towards determining the appropriate methodology will be demonstrated in the reference section of a paper. However, the results of this study indicate that research into the most appropriate methods and/or the referencing of the quantitative sources discussing these methods, is not showing up in the reference section of psychological studies.

\section{Study Two}

This study investigated the degree to which quantitative methodology articles are being referenced by nonquantitative researchers and by fellow quantitative methodology researchers. The second study approached the research question from a different direction than the first study. Whereas Study One looked at non-quantitative articles and determined the number of quantitative references, Study Two looked at quantitative articles (henceforth referred to as target articles) and determined whether they were being referenced in other quantitative literature or in non-quantitative literature. Specifically, the study aimed to compare the rate of references to the target articles in quantitative methodology literature to those in non-quantitative literature.

\section{Method}

Four journals that primarily publish articles that focus on quantitative methods were utilized: Psychometrika (PMET), British Journal of Mathematical and Statistical Psychology (BJMSP), Journal of Educational and Behavioral Statistics (JEBS), and Psychological Methods (PM). These journals were selected because they publish articles on quantitative methods that are intended to provide psychology researchers with improved and novel methods for analyzing their data.

The Institute for Scientific Information's (ISI's) Web of Science was used to determine the number of times each target article was referenced, and a review of the referencing article allowed for the determination of whether the referencing publication was quantitative or non-quantitative in nature.

The publication years that were selected were 1993, 1994, 
Table 2. Measures of central tendency for the total number of references and for the proportion of the total references that were quantitative, across each journal.

\begin{tabular}{lllllllll}
\hline Journal & & \multicolumn{3}{c}{ Total References } & \multicolumn{4}{c}{$\begin{array}{c}\text { Proportion of the Total Number of } \\
\text { References that were quantitative }\end{array}$} \\
\hline & Mode & Med & Mean & TrMean & Mode & Med & Mean & TrMean \\
\hline & & & & & & & & \\
JPSP & 65 & 66.5 & 68.9 & 67.8 & $0 \%$ & $2.1 \%$ & $3.4 \%$ & $2.8 \%$ \\
PB & 23 & 201.5 & 198.5 & 197.2 & $0 \%$ & $0.5 \%$ & $3.5 \%$ & $1.6 \%$ \\
CD & 59 & 56 & 59.1 & 58.1 & $0 \%$ & $0 \%$ & $2.6 \%$ & $1.8 \%$ \\
JCCP & 45 & 46 & 47.7 & 46.6 & $0 \%$ & $3.6 \%$ & $5.2 \%$ & $4.6 \%$ \\
JAP & 64 & 64 & 73.9 & 67.7 & $0 \%$ & $3.8 \%$ & $5.2 \%$ & $4.8 \%$ \\
IJOP & 43 & 44 & 49.2 & 46.1 & $0 \%$ & $0 \%$ & $1.2 \%$ & $0.8 \%$ \\
\hline
\end{tabular}

Note: Med = Median; TrMean = 5 \% Trimmed Mean; JPSP = Journal of Personality and Social Psychology; PB = Psychological Bulletin; CD = Child Development; JCCP = Journal of Consulting and Clinical Psychology; JAP = Journal of Applied Psychology; IJOP = International Journal of Organizational Psychology.

2003 and 2004. These years were selected because they include recent articles, but are not so recent that they have not had adequate time to be 'discovered' and referenced. All articles within the journal were included unless they were a commentary on other published articles. For each target article, the following information was recorded: 1) the number of times it was referenced; 2) whether the referencing sources were quantitative or non-quantitative in nature; 3) whether the referencing sources were published by the same author as the target article; and 4) whether the referencing sources that were published by the same author as the target article were quantitative or non-quantitative in nature. As with Study One, for any article in which there was ambiguity about the coding, the decision was made jointly by the authors.

In total, 394 articles were reviewed from the 1993, 1994, 2003, and 2004 publishing years of the journals BJMSP, PMET, JEBS and PM. The only exception was the fourth issue of JEBS in 1994, which was not included in the ISI Web of Science database and thus the articles from this issue were excluded. The years 1993 and 1994 were collapsed (and referred to as the 1990s) and the years 2003 and 2004 were likewise collapsed (and referred to as the 2000s). For each journal in each time category, Table 2 presents the total number of articles reviewed, the median number of quantitative referencing sources, the median number of nonquantitative referencing sources, and the median number of references to the target article (quantitative and nonquantitative) after removing references that had the same author as the target article. It is important to point out that the distributions of the outcome variables were severely positively skewed, and therefore the median results were chosen as the most representative measure of central tendency. Other measures of central tendency are given in parentheses.

\section{Results and Discussion}

\section{Summary of Articles Reviewed}

For articles published in the 1990s, the target articles were referenced in other quantitative methodology sources a median of 6 times $(\mathrm{M}=9.93 ; 5 \%$ trimmed mean = 8.07; Mode $=0)$, and in non-quantitative sources a median of 2 times ( $\mathrm{M}$ $=18.63 ; 5 \%$ trimmed mean $=7.16 ;$ Mode $=0$ ). For articles published in the 2000s, the target articles were referenced in other quantitative methodology sources a median of 1 time $(\mathrm{M}=2.01 ; 5 \%$ trimmed mean $=1.60$; Mode $=0)$, and in nonquantitative sources a median of 0 times $(\mathrm{M}=2.27 ; 5 \%$ trimmed mean $=1.50$; Mode $=0$ ). Thus, after over fifteen years in circulation, a paper in a quantitative methodology journal can expect to be referenced only a few times by nonquantitative researchers. Much more likely is the prospect of being referenced by fellow quantitative researchers, as the median number of references is three times that of nonquantitative scientists.

\section{Differences in Referencing Across Years}

To determine whether there was a difference between time categories in the number of references, the Welch test on ranked data (Cribbie, Wilcox, Bewell \& Keselman, 2007) was employed to accommodate the skewed and heteroscedastic nature of the data. For the number of nonquantitative sources referencing the target article, there were significantly more references from the 1990s (Median $=2$ ) than from the 2000s (Median $=0), \mathrm{t}_{\mathrm{w}}(321.26)=5.56, \mathrm{p}<.001$, $\eta^{2}=.088$ (moderate effect size). For the number of 
Table 3. Median Number of Quantitative and Non-quantitative Referencing Articles per Journal and Year

\begin{tabular}{lllllll}
\hline Year & Journal & \# Articles & QUANT & $\begin{array}{l}\text { QUANT } \\
\text { w/o SA }\end{array}$ & NQ & $\begin{array}{l}\text { NQ } \\
\text { w/o SA }\end{array}$ \\
\hline 1993 & BJMSP & 19 & 3.0 & 2.0 & 1.0 & 1.0 \\
& PMET & 34 & 6.0 & 4.0 & 2.0 & 1.5 \\
& JEBS & 16 & 6.0 & 5.0 & 3.5 & 2.0 \\
& PM & 14 & 16.0 & 14.5 & 59.0 & 58.0 \\
\hline 1994 & BJMSP & 17 & 10.0 & 6.0 & 1.0 & 1.0 \\
& PMET & 36 & 4.0 & 3.0 & 1.0 & 1.0 \\
& JEBS & 17 & 6.0 & 3.0 & 1.0 & 1.0 \\
& PM & 10 & 8.0 & 6.5 & 12.5 & 12.5 \\
\hline 2003 & BJMSP & 21 & 2.0 & 0.0 & 1.0 & 0.0 \\
& PMET & 26 & 1.0 & 0.0 & 1.0 & 0.0 \\
& JEBS & 26 & 0.0 & 0.0 & 0.5 & 0.0 \\
& PM & 46 & 1.0 & 0.0 & 3.0 & 2.0 \\
\hline 2004 & BJMSP & 21 & 1.0 & 0.0 & 0.0 & 0.0 \\
& PMET & 27 & 1.0 & 0.0 & 0.0 & 0.0 \\
& JEBS & 35 & 0.0 & 0.0 & 0.0 & 0.0 \\
& PM & 29 & 2.0 & 2.0 & 2.0 & 2.0 \\
\hline All & BJMSP & 78 & 2.0 & 1.0 & 0.5 & 0.0 \\
Years & PMET & 123 & 3.0 & 1.0 & 1.0 & 0.0 \\
& JEBS & 94 & 1.0 & 0.0 & 1.0 & 0.0 \\
& PM & 99 & 3.0 & 2.0 & 4.0 & 4.0 \\
\hline
\end{tabular}

Note: \# Articles = number of articles evaluated; QUANT = quantitative methodology articles referencing the target article; QUANT w/o SA = quantitative methodology articles referencing the target article, after removing statistical articles with the same author as the target quantitative article; $N Q=$ non-quantitative articles referencing the target article; NQ w/o SA = non-quantitative articles referencing the target article, after removing applied articles with the same author as the target article; BJMSP $=$ British Journal of Mathematical and Statistical Psychology; PMET = Psychometrika; JEBS = Journal of Educational \& Behavioral Statistics; PM = Psychological Methods.

quantitative methodology sources referencing the target article, there were significantly more references from the 1990s (Median =6) than from the 2000s (Median = 1), $\mathrm{t}_{\mathrm{w}}(338.93)=11.47, \mathrm{p}<.001, \eta^{2}=.279$.

\section{Differences in the Frequency of Quantitative and Non- Quantitative References}

In order to determine if there are differences in the frequency of quantitative and non-quantitative references to the target articles, we compared the number of references that were non-quantitative versus the number that were quantitative across all journals and years using a paired samples $t$ test. In order to minimize the impact of extreme cases, any case with a z-score greater than 3 (in absolute value) on the difference between quantitative and nonquantitative references was removed. The remaining difference scores were approximately normally distributed. Target articles were referenced more in quantitative methodology sources $(\mathrm{M}=4.16, \mathrm{~s}=6.21)$ than in nonquantitative sources $(\mathrm{M}=3.32, \mathrm{~s}=6.24), \mathrm{t}(379)=2.14, \mathrm{p}=$
$.033, \eta^{2}=.012$, although the effect size is very small.

A paired t-test was also used to investigate differences in non-quantitative and quantitative methodology sources that were authored by the same individual that published the target article. As expected, there was a significantly greater number of quantitative methodology references than nonquantitative references with the same author as the target quantitative methods article, $\mathrm{t}(393)=5.95, \mathrm{p}<.001, \eta^{2}=.29$. For both quantitative and non-quantitative referencing sources, the median number of same-author references was 0 . The maximum number of non-quantitative same-author references was 8 while for quantitative same-author references was 25, while the variances were 1.51 and 5.40, respectively. After removing articles that had the same author as the target article, there was a significant, but small, difference between the number of non-quantitative sources (Median =1) and quantitative methodology sources (Median $=1$ ) referencing the target articles, $\mathrm{t}(393)=2.297, \mathrm{p}=.026, \eta^{2}=$ .013 . 


\section{Differences in Referencing Across Journals}

The last four rows of Table 3 present the median number of quantitative and non-quantitative sources referencing the target article for each journal, across all four years. An important consideration is whether there are differences in the number of quantitative or non-quantitative sources referencing the target article across journals. Again, given that each of the outcome measures was severely positively skewed, all analyses were conducted on the ranks of the original data. Further, to account for any differences in the variances of the outcome measures, after ranking, across the journals (BJMSP, PMET, JEBS, PM), Welch's omnibus (and Games-Howell pairwise comparisons) were used. There were significant differences across the journals in terms of the number of non-quantitative sources referencing the target article, $F_{w}(3,209.29)=15.47, p<.001$. Specifically, there were significantly more non-quantitative sources referencing target articles in PM than in BJMSP $(p<.001)$, PMET $(p<.001)$ or JEBS $(p<.001)$. There were no significant differences between BJMSP and PMET ( $\mathrm{p}=.535)$, BJMSP and JEBS ( $p=.791)$, or PMET and JEBS $(p=.981)$.

There were significant differences across the journals in terms of the number of quantitative methodology sources referencing the target article, $\mathrm{F}_{\mathrm{w}}(3,207.27)=3.61, \mathrm{p}=.014$. There were fewer quantitative methodology sources referencing the target article from JEBS than from PM ( $p=$ $.050)$ or PMET $(p=.011)$. However, there were no significant differences between PM and BJMSP ( $\mathrm{p}=.860)$, PM and PMET $(\mathrm{p}=.993)$, BJMSP and PMET $(\mathrm{p}=.668)$, or BJMSP and JEBS ( $\mathrm{p}$ $=.265)$.

To summarize the results of Study 2, consider that even after about fifteen years, quantitative methodology researchers can expect that their publications in popular quantitative methods sources will be referenced about two times in non-quantitative research publications. Even more disturbing is that the modal number of references in nonquantitative studies, for articles published in quantitative methodology journals about 15 years ago, is zero. Further, for studies published about 5 years ago, both the mode and median number of references in non-quantitative studies is zero. This is not to say that if you publish a quantitative methods article in a quantitative methods journal that the likelihood of the article being widely read and cited is nil; we found a few quantitative methods articles in this study from the 1990s that had been cited more than 200 times. However, from a purely probabilistic standpoint, the likelihood of a great number of citations is pretty small.

The results of Study 2 also verify some intuitive hypotheses. First, the amount of time an article has been available to researchers significantly increases the number of citations to that article (i.e., researchers are not just citing recent articles and then forgetting about them). Second, authors of quantitative methodology articles tend to reference these articles more in other quantitative methodology sources than in non-quantitative sources.

One very interesting result from Study 2 (especially for authors of quantitative methodology publications) is that articles published in the journal 'Psychological Methods' have a much greater probability of being cited by nonquantitative researchers than articles published in any of the other quantitative methodology journals. For example, quantitative methods articles published in PM in 1993 or 1994 had a median of 24 citations in non-quantitative sources, whereas the median number of citations for articles published in JEBS, PMET or BJMSP in the same years did not exceed two.

\section{Overall Discussion}

Together, the results of these studies imply that the authors of psychological studies pay little attention to published articles dealing with advances in quantitative methods. For example, Study 1 found that the median and modal number of references to quantitative methodology sources in non-quantitative articles were one and zero, respectively. In Study 2 it was found that, for quantitative methods studies published about 15 years ago, the median and modal number of citations in non-quantitative research articles was 2 and 0 , respectively. It is important to point out that we were not expecting an extremely large number of references to quantitative methodology research; however the results of this study indicate that the number of references to quantitative methodology research is so small that we believe that there is need for concern.

The studies were not without limitations. Although we believe that the findings of this study (based on the specific journals chosen) would be consistent across other journals, it is possible that this is not the case. Further, although we believe that the years selected for this study provide a robust look at recent referencing patterns, it is possible that different results might be obtained with different publication years. These issues might be addressed in future research. For example, it would be beneficial to compare the rates of citation to substantive (non-quantitative) research articles to the rates of citation to quantitative methodology research articles in psychological literature. In other words, such a study would determine whether substantive articles receive more or less citations than quantitative methodology articles, regardless of the quality of the journal in which the research was cited.

The obvious rebuttal to the results of this study, as discussed above, is that researchers are utilizing quantitative methodology sources in deriving their data analysis 
strategy, but are simply not referencing these sources. Not only do we find this rebuttal implausible due to the fact that psychologists are generally rigorous in referencing but also because it has been noted in previous literature that researchers are routinely utilizing inappropriate statistical procedures in analyzing their data. In fact, Keselman et al. (1998) concluded that "substantive researchers need to wake up both to the (inappropriate) statistical techniques that are currently being used in practice and to the (more appropriate) ones that should be being used" (p. 380). However, if this is in fact the case, then we hope that this paper can be used as a reminder to researchers to be sure to reference the quantitative methodology sources they utilize for researching the most appropriate data analytic approaches.

With the social, political, and health implications of many psychological studies, it is of utmost importance that the results and conclusions of the studies be as accurate as possible. Thus, we encourage researchers to be diligent in researching, selecting and referencing the most appropriate statistical techniques for their research questions and data characteristics. In many cases it is possible to gain information about the most appropriate data analytic strategy from up-to-date textbooks, although typically the most accurate and extensive recommendations for conducting appropriate statistical analyses will be found in articles published in quantitative methods journals. Further, we believe it is imperative that journal editors also play an important role in ensuring that the research hypotheses from psychological studies are analyzed properly. Specifically, we recommend that at least one reviewer of each manuscript be competent in assessing the appropriateness of the statistical methods adopted for testing the research hypotheses, and, where appropriate, that editors/reviewers encourage authors to explore, utilize and reference modern/improved methods for addressing their research questions. Finally, as suggested by an anonymous reviewer, we recommend that quantitative methodology articles are included in non-quantitative journals at regular intervals (e.g., every two years). For example, special issues of the journals could highlight recent advances in methodological approaches relevant to the field of research. This would help to ensure that discussions regarding novel and appropriate methodological approaches are readily available to researchers. With these recommendations in mind, it is our hope that researchers become more cognizant of the most appropriate strategies for analyzing psychological data.

\section{References}

Adair, J. G. \& Vohra, N. (2003). The explosion of knowledge, references, and citations. American Psychologist, 58, 15-23.

Bradley, J. V. (1977). A common situation conducive to bizarre distribution shapes. The American Statistician, 31, 147-150.

Cribbie, R. A., Wilcox, P. R., Bewell, C. \& Keselman, H. J. (2007). Tests for treatment group equality when data are nonnormal and heteroscedastic. Journal of Modern Applied Statistical Methods, 6, 117-132.

Cumming, G., Fidler, F., Leonard, M., Kalinowski, P., Christiansen, A., Kleinig, A., Lo, J., McMenamin, N., \& Wilson, S. (2007). Statistical reform in psychology: Is anything changing? Psychological Science, 18, 230-232.

Erceg-Hurn, D. M. (2008). Modern robust statistical methods. An easy way to maximize the accuracy and power of your research. American Psychologist, 63, 591601.

Games, P. A. \& Howell, J. F. (1976). Pairwise multiple comparison procedures with unequal $\mathrm{Ns}$ and/or variances: A Monte Carlo study. Journal of Educational Statistics, 1, 113-125.

Golinski, C. \& Cribbie, R. A. (in press). The expanding role of quantitative methodologists in advancing psychology. Canadian Psychology.

Grissom, R. J. (2000). Heterogeneity of variance in clinical data. Journal of Consulting and Clinical Psychology, 68, 155165.

Keselman, H. J., Huberty, C. J., Lix, L. M., Olejnik, S., Cribbie, R., Donahue, B., Kowalchuk, R. K., Lowman, L. L., Petoskey, M. D., Keselman, J. C. \& Levin, J. R. (1998). Statistical practices of educational researchers: An analysis of their ANOVA, MANOVA, and ANCOVA analyses. Review of Educational Research, 68, 350-386.

Micceri, T. (1989). The unicorn, the normal curve, and other improbable creatures. Psychological Bulletin, 105, 156-166.

Moed, H. F. (2005). Citation analysis in research evaluation. Dordrecht, The Netherlands: Springer.

Reiss, H. T. \& Stiller, J. (1992). Publication trends in JPSP: A three-decade review. Personality and Social Psychology Bulletin, 18, 465-472.

Safer, M. A. \& Tang, R. (2009). The psychology of referencing in psychology journal articles. Issues in Publishing, Editing, and Reviewing, 5, 51-53.

Thorngate, W. (1990). The economy of attention and the development of psychology. Canadian Psychology, 31, 262-271.

Welch, B. L. (1951). On the comparison of several mean values: An alternative approach. Biometrika, 38, 330-336.

Wilkinson, L. \& TFSI (1999). Statistical methods in 
Psychology journals: Guidelines and explanations. American Psychologist, 54, 594-604.

Wilcox, R.R. (2002). Can the weak link in psychological research be fixed? Association for Psychological Science: Observer, 15. Retrieved April 10, 2008, from http://www.psychologicalscience.org/observer/getArticle .cfm?id=931.
Wilcox, R. R. \& Keselman, H. J. (2003). Modern robust data analysis methods: Measures of central tendency. Psychological Methods, 8, 254-274.

Zumbo, B. D., \& Jennings, M. J. (2002). The robustness of validity and efficiency of the related samples t-test in the presence of outliers. Psicologica: Revista de Metodología y Psicología Experimental, 23, 415-450.

Manuscript received June 11 2009.

Manuscript accepted September 28 $8^{\text {th }}, 2010$. 